\title{
Melanoma Cutâneo: Perfil Epidemiológico dos Óbitos no Estado de São Paulo - Brasil entre 2005 e 2014
}

\author{
Veridiana de Paula Santos Miranda', Flávia Regina Ferreira², Andrea Paula Peneluppi de Medeiros ${ }^{3}$ \\ 'Dermatologia, Hospital Heliópolis, São Paulo, Brasil \\ ${ }^{2}$ Dermatologia, Departamento de Medicina, Universidade de Taubaté/ Serviço de Dermatologia, Hospital Universitário de Taubaté - \\ HUT/UNITAU, Taubaté, Brasil \\ ${ }^{3}$ Saúde Coletiva/ Ciências Ambientais, Departamento de Medicina, Universidade de Taubaté, Taubaté, Brasil
}

RESUMO - Introdução: O melanoma cutâneo $(M C)$ é o cancro de pele menos frequente, porém, responsável pela maioria dos óbitos. Este estudo teve como objetivo avaliar o perfil epidemiológico dos óbitos por MC no Estado de São Paulo, bem como avaliar os fatores que podem determinar a sua ocorrência segundo o sexo. Métodos: Foram estudados dados relativos à mortalidade por MC no Estado de São Paulo-Brasil entre 2005 e 2014 . Foram realizadas análises descritivas e regressão logística univariada e multivariada para determinar a probabilidade de óbito quanto ao sexo segundo variáveis demográficas. A análise estatística baseou-se no cálculo da OR. Em todas as análises foram considerados intervalos de confiança de $95 \%$ e adotado nível de significância de alfa $=5 \%$. Resultados: Os 4059 óbitos por MC no período representaram 0,9\% da mortalidade geral por neoplasia. Destes, $56,7 \%$ referiam-se ao sexo masculino e $92,4 \%$ à raça branca. A idade média no momento do óbito foi 63,5 anos (DP=16,3). Na regressão logística multivariada, a ausência de parceiro e o maior nível de escolaridade mostraram-se fatores discordantes entre os sexos. Conclusão: Este estudo permitiu avaliar o perfil epidemiológico dos óbitos por melanoma, sendo possível identificar o predomínio de indivíduos do sexo masculino, da raça branca, com parceiros (as), com baixa escolaridade e uma tendência ao aumento do número de óbitos nos últimos 5 anos do período estudado. Além disso, pôde-se verificar diferenças nos fatores de risco relacionadas ao sexo dos indivíduos.

PALAVRAS-CHAVE - Brasil; Melanoma/epidemiologia; Melanoma/mortalidade; Neoplasias da Pele/epidemiologia; Neoplasias da Pele/mortalidade.

\section{Cutaneous Melanoma: Epidemiological Profile of Deaths in the State of São Paulo - Brazil between 2005 and 2014}

ABSTRACT - Introduction: Cutaneous melanoma (CM) is the least common skin cancer, but it accounts for the majority of deaths. This study aimed to evaluate the epidemiological profile of deaths due to CM in the State of São Paulo, as well as to evaluate the factors that may determine their occurrence by gender. Methods: Data on CM mortality in the State of São Paulo, Brazil, between 2005 and 2014 were studied. Descriptive analyzes and univariate and multivariate logistic regression were performed to determine the probability of death in terms of gender according to demographic variables. Statistical analysis was based on OR calculation. In all analyzes, 95\% confidence intervals were used and alpha $=5 \%$ level of significance was adopted. Results: During the study period 4059 deaths due to CM were identified, representing $0.9 \%$ of the general mortality due to neoplasia. Of these, $56.7 \%$ were male and $92.4 \%$ were white. The mean age at death was 63.5 years $(S D=16.3)$. In the multivariate logistic regression, the absence of partner and the higher level of education showed to be discordant factors between genders. Conclusion: This study evaluating the epidemiological profile of deaths due to $\mathrm{CM}$, identified the predominance of the male gender, Caucasians and individuals with partners and a low level of education. There was a trend towards an increase in the number of deaths in the last 5 years of the study period. In addition, we observed differences in risk factors related to the gender.

KEYWORDS - Brazil; Melanoma/epidemiology; Melanoma/mortality; Skin Neoplasms/epidemiology; Skin Neoplasms/mortality.

Correspondência: Veridiana de Paula Santos Miranda Avenida Dom Duarte Leopoldo e Silva, 625, Apto 66

Taubaté, São Paulo, Brasil - CEP: 12070-590

E-mail: veridiana.santos@outlook.com

DOI: https://dx.doi.org/10.29021/spdv.76.4.923
Recebido/Received

23 Março/March 2018

Aceite/Accepted

01 Novembro/November 2018 


\section{INTRODUÇÃO}

melanoma cutâneo (MC) é uma neoplasia de pele, originária da proliferação anormal e desordenada dos melanócitos. ${ }^{1}$ Entre as neoplasias cutâneas, o MC é a entidade menos frequente, representando $4 \%$ a $6 \%$ destas neoplasias, porém o responsável pela maioria dos óbitos. ${ }^{2}$

Os principais fatores de risco para o desenvolvimento do MC resultam da combinação de fatores constitucionais/genéticos e ambientais, dos quais os principais são o fototipo e a exposição à radiação ultravioleta, particularmente quando esta ocasiona queimaduras graves. ${ }^{3-5}$ A presença e o número de nevos melanocíticos congênitos e adquiridos, comuns ou displásicos, são também fatores de risco constitucionais importantes em pessoas de pele clara. ${ }^{1,3}$

As maiores taxas de incidência do $M C$ em todo o mundo têm sido relatadas na Austrália e Nova Zelândia, com média de 51,6 e 51,8 casos novos/100 000 habitantes, respectivamente, entre 2007 e 2011 1.6,7 Na América Latina observam-se taxas intermediárias a baixas. Entretanto, as menores taxas de incidência do $M C$ encontram-se nos continentes Asiático e Africano, com menos de 2 casos novos/100 000 habitantes em 2015.8

No Brasil, as estimativas para o ano de 2018, segundo - Instituto Nacional do Câncer (INCA), são de 6260 casos novos de MC, sendo 2920 para homens e 3340 para mulheres. As menores taxas estimadas encontram-se na região norte e as maiores na região sul do país.9,10 Para o Estado de São Paulo, neste mesmo ano, foram estimados 740 casos novos no sexo masculino (incidência de 3,27 casos novos/100 000 habitantes) e 1050 casos novos no sexo feminino (incidência de 3,17 casos novos/100 000 habitantes), representando um importante problema de saúde pública. ${ }^{9}$

Ao contrário de outros tipos de cancro cutâneo, o MC frequentemente afeta indivíduos mais jovens, com uma idade média de cinquenta anos, pelo que está entre os cancros mais graves quando avaliado de acordo com o número de anos de vida perdidos. Além disso, apesar de todos os esforços e o desenvolvimento de novas terapias em casos avançados, ainda não se alcançou a cura ou melhoria significativa nas taxas de sobrevivência. ${ }^{11}$

Diante do exposto, torna-se evidente a importância de estudar as características epidemiológicas relacionadas à mortalidade por MC. O conhecimento do perfil desses pacientes pode contribuir para uma melhor compreensão deste tumor e de seu comportamento, fornecendo dados aos gestores de saúde para o planejamento de intervenções (medidas de prevenção primária e secundária) que possibilitem impactar na sua morbimortalidade, o que motivou este estudo.

O presente estudo teve como objetivo avaliar o perfil epidemiológico dos óbitos por melanoma cutâneo no Estado de São Paulo, Brasil, num período de dez anos.

\section{MÉTODOS}

Trata-se de um estudo retrospectivo, com dados relativos à mortalidade por $M C$ no Estado de São Paulo no período de
2005 a 2014, obtidos no portal eletrônico do Departamento de Informação e Informática do Sistema Único de Saúde (DATASUS), ${ }^{12}$ por meio do Sistema de Informação sobre Mortalidade (SIM). Para obtenção dos dados foi necessário realizar a transferência dos arquivos por meio do programa Tabwin ${ }^{\circledR}$ disponibilizado pelo site. Neste portal, os óbitos encontram-se classificados de acordo com a $10^{a}$ Conferência de Revisão da Classificação Internacional de Doenças (CID-10), tendo sido selecionados para este estudo somente os óbitos com causa primária por melanoma da pele.

A variável dependente foi o óbito por $M C$ e as variáveis independentes contempladas foram: idade (anos), sexo (masculino ou feminino), raça (branca ou não branca), estado civil, município de residência e nível de escolaridade (anos de estudo), Para fins de estudos demográficos, a atual classificação racial do IBGE (Instituto Brasileiro de Geografia e Estatística) tomada como oficial no Brasil, desde 1991 inclui as seguintes categorias: branco, preto, pardo, amarelo e indígena. ${ }^{4}$ Foram criadas novas variáveis a partir da recodificação das já existentes. Para caracterizar o estado civil do paciente foram criados com base na categoria inicial 2 grupos - com parceiro (a) e sem parceiro (a) - e para a escolaridade 4 categorias: nenhum ano de estudo concluído, 1 a 7 anos de estudo concluídos, 8 a 11 anos de estudo concluídos e 12 ou mais anos de estudo concluídos.

Primeiramente, realizou-se a análise descritiva das variáveis selecionadas para o estudo. A partir destas análises, foram criadas tabelas e gráficos para melhor exposição dos dados. Posteriormente, foi realizado uma análise estatística baseada na regressão logística univariada com cálculo da odds ratio (OR) para se estimar a probabilidade do óbito por melanoma cutâneo no sexo masculino, comparado ao sexo feminino, associando-se a cada variável. Após esta análise, houve o ajustamento para as seguintes variáveis: estado civil e escolaridade. Foram considerados intervalos de confiança de 95\% e adotado nível de significância de 5\% ( $\alpha=5 \%$ ). Utilizou-se para a análise o Programa STATA versão $9 .{ }^{13}$

\section{RESULTADOS}

No período de 10 anos do estudo (2005-14) foram obtidas do portal DATASUS 4059 informações sobre óbitos por melanoma cutâneo, representando $0,9 \%$ da mortalidade geral por neoplasia no Estado de São Paulo. Destas, 2303 $(56,7 \%)$ referiam-se ao sexo masculino e 3568 (92,4\%) à raça branca. A descrição das variáveis e informações sobre estado civil e escolaridade podem ser observadas na Tabela 1.

A idade média no momento do óbito foi de 63,5 anos (desvio padrão $(D P)=16,3$ ), variando de 12 a 99 anos. A idade média no sexo masculino foi de 63,1 anos (DP $=$ 15,4 ) e no sexo feminino, 64,5 anos (DP $=16,6$ ). Quanto à faixa etária, houve um predomínio da mortalidade entre 50 e 79 anos, representando 62,3\% dos óbitos. Resultado semelhante foi observado quando analisados os sexos separadamente (Fig. 1).

Ao avaliar o número total de óbitos por ano durante o período do estudo, observa-se uma tendência ao aumento 
Tabela 1 - Distribuição dos óbitos por melanoma segundo sexo, raça, estado civil e escolaridade. Estado de São Paulo, Brasil, 2005-2014.

\begin{tabular}{|c|c|c|}
\hline Variáveis & $\stackrel{N}{(N \text { Total=4059) }}$ & $\%$ \\
\hline $\begin{array}{l}\text { Sexo } \\
\text { - Masculino } \\
\text { - Feminino }\end{array}$ & $\begin{array}{l}2303 \\
1756\end{array}$ & $\begin{array}{l}56,7 \\
43,3\end{array}$ \\
\hline $\begin{array}{l}\text { Raça } \\
\text { - Branca } \\
\text { - Preta } \\
\text { - Amarela } \\
\text { - Parda }\end{array}$ & $\begin{array}{c}3568 \\
77 \\
27 \\
191\end{array}$ & $\begin{array}{l}92,4 \\
2,0 \\
0,7 \\
4,9\end{array}$ \\
\hline $\begin{array}{l}\text { Estado Civil } \\
\text { - Com parceiro } \\
\text { (a) } \\
\text { - Sem parceiro } \\
\text { (a) }\end{array}$ & $\begin{array}{l}2272 \\
1596\end{array}$ & $\begin{array}{l}58,7 \\
41,3\end{array}$ \\
\hline $\begin{array}{l}\text { Escolaridade } \\
\text { (anos de estudos } \\
\text { concluídos) } \\
\text { - Nenhum } \\
\text { - } 1 \text { a } 7 \\
\text { - } 8 \text { a } 11 \\
\text { - } 12 \text { ou mais }\end{array}$ & $\begin{array}{c}308 \\
1837 \\
653 \\
606\end{array}$ & $\begin{array}{l}9,0 \\
54,0 \\
19,2 \\
17,8\end{array}$ \\
\hline
\end{tabular}

Nota: 196, 191 e 655 casos sem registros, respectivamente, para as variáveis raça, estado civil e escolaridade.

da mortalidade por melanoma no Estado de São Paulo nos últimos cinco anos, sendo o número de óbitos entre 2005 e 2009 de 1892 e entre 2010 e 2014 de 2167 . O número de óbitos por ano durante o período do estudo e segundo o sexo pode ser observado no Fig. 2. A taxa de mortalidade para o período do estudo foi de 0,92 e 1,03 por 100000 habitantes para os intervalos de anos entre 2005 e 2009 e 2010 a 2014 , respectivamente. ${ }^{4}$

Realizou-se regressão logística univariada e, após ajustes com as variáveis que apresentaram significância estatística (estado civil e escolaridade), foi realizada a regressão logística multivariada (Tabela 2). Em relação ao estado civil e nível de escolaridade, a ausência de um parceiro mostrou-se um fator de proteção e o maior nível de escolaridade mostrou-se um fator de risco no sexo masculino, mesmo após os ajustes na análise multivariada. No sexo feminino, a ausência de parceiro indicou maior probabilidade de óbito por $M C$ e o maior nível de escolaridade mostrou-se como fator protetor, mantendo-se também após os ajustes na análise multivariada.

\section{DISCUSSÃO}

O presente estudo permitiu identificar o perfil epidemiológico relacionado à mortalidade por $\mathrm{MC}$ no Estado de São Paulo, entre 2005 e 2014, fornecendo dados originais e subsidiando uma melhor compreensão deste tumor e de seu comportamento, possibilitando futuras intervenções e norteando tomadas de decisão.

Representando 0,9\% da mortalidade por neoplasia no Estado e São Paulo, a maioria dos óbitos por MC ocorreu no sexo masculino. Esse predomínio foi observado praticamente em todas as faixas etárias sendo consistente com a literatura internacional. ${ }^{14-16} \mathrm{Na}$ literatura nacional os dados são controversos. ${ }^{17,18}$ Ferreira et a ${ }^{19}$ em estudo recente sobre mortalidade por MC no Sul do Brasil identificaram uma maior mortalidade para o sexo masculino, concordando com o presente estudo. Entretanto, Pinto et a ${ }^{21}$ e Martins-Costa et $a^{22}$ em seus estudos observaram um predomínio da mortalidade no sexo feminino. As diferenças comportamentais

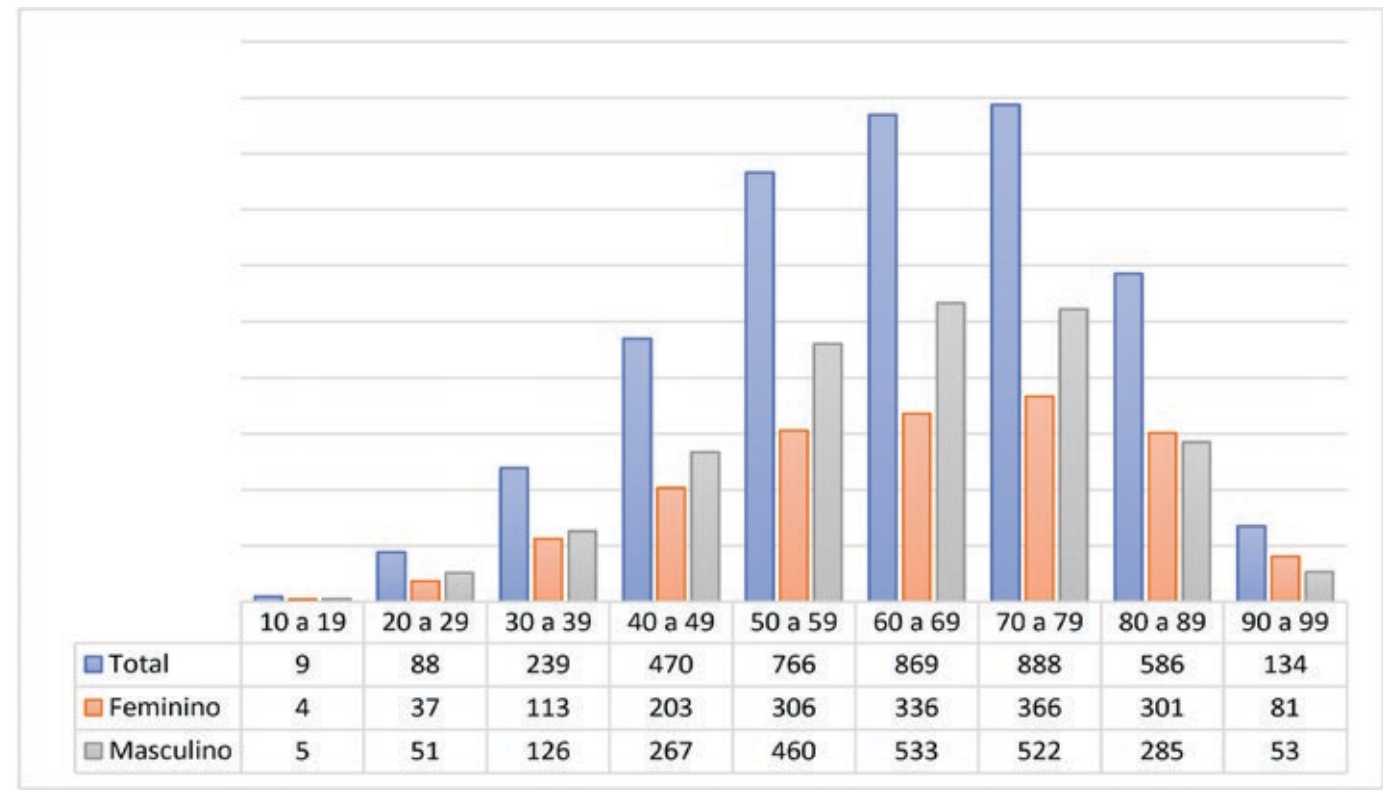

Figura 1 - Distribuição dos óbitos por melanoma segundo a faixa etária e o sexo. Estado de São Paulo, Brasil, 2005-2014. 


\section{Artigo Original}

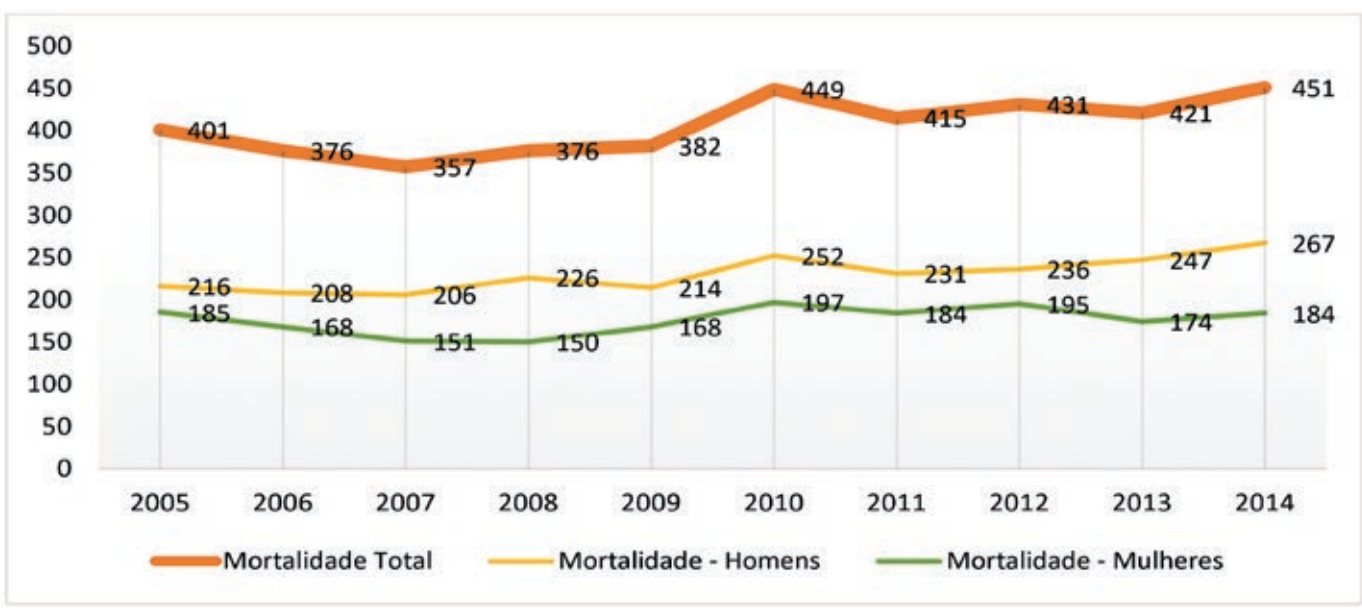

Figura 2 - Distribuição dos óbitos por melanoma cutâneo em números absolutos e por sexo. Estado de São Paulo, Brasil, $2005-2014$.

Tabela 2 - Valores das odds ratio (OR) e respectivos intervalos de confiança (IC 95\%) e valores de p para os óbitos por melanoma por sexo, segundo características epidemiológicas (regressão logística univariada e multivariada). Estado de São Paulo, Brasil, 2005-2014.

\begin{tabular}{|c|c|c|c|c|}
\hline & \multicolumn{2}{|c|}{ Regressão Logística Univariada } & \multicolumn{2}{|c|}{ Regressão Logística Multivariada } \\
\hline Variáveis & OR / IC 95\% & OR / IC 95\% & OR / IC 95\% & OR / IC 95\% \\
\hline \multicolumn{5}{|l|}{ Faixa etária } \\
\hline - 1 a 39 anos & 1 & 1 & - & - \\
\hline \multirow[t]{2}{*}{ - $>60$ anos } & $1,09(0,86-1,37)$ & $0,92(0,73-1,16)$ & - & - \\
\hline & $p=0,76$ & $p=0,76$ & - & - \\
\hline \multicolumn{5}{|l|}{ Estado Civil } \\
\hline - Com parceiro (a) & 1 & 1 & 1 & 1 \\
\hline - Nenhuma & 1 & 1 & 1 & 1 \\
\hline - 1 a 11 anos & $1,49(1,18-1,89)$ & $0,67(0,53-0,85)$ & $1,35(1,05-1,76)$ & $0,74(0,57-0,95)$ \\
\hline \multirow[t]{2}{*}{ - > 12 anos } & $2,06(1,57-2,73)$ & $0,48(0,36-0,64)$ & $1,71(1,25-2,32)$ & $0,59(0,43-0,80)$ \\
\hline & $p=0,005$ & $p=0,005$ & $p=0,005$ & $p=0,005$ \\
\hline \multicolumn{5}{|l|}{ Raça } \\
\hline - Branca & 1 & 1 & - & - \\
\hline - Preta & $1,00(0,64-1,58)$ & $0,99(0,63-1,58)$ & - & - \\
\hline
\end{tabular}


entre os sexos (auto-exame, procura por atendimento médi$\mathrm{co}$, aderência às orientações quanto à fotoproteção) podem justificar este achado e as diferenças quanto ao tipo de estudo (base populacional/base hospitalar), podem explicar, pelo menos em parte, as discordâncias encontradas. A predominância da mortalidade por $M C$ em mulheres em regiões geográficas com baixos índices de luz solar, diferente das condições encontradas no Brasil e, mais especificamente, no Estado de São Paulo também já foi reportada. ${ }^{20}$

Aspectos étnicos exercem influência nas características clínicas, patológicas, localização anatómica e prognóstico do MC. ${ }^{21,22}$ No presente estudo, mais de $90 \%$ dos óbitos ocorreram na raça branca, seguida pela raça parda. Como fatores de confusão da análise, considera-se, a grande diversidade étnica em nosso país, sendo difícil encontrar traços exclusivos de uma determinada etnia na população geral e a menor acessibilidade aos serviços de saúde por parte de algumas etnias específicas.

No que se refere ao estado civil, mais da metade dos indivíduos deste estudo possuíam parceiros no momento do óbito. No sexo masculino, a ausência de parceiro mostrou-se como fator de proteção e no sexo feminino, como fator de risco. Este dado merece especial atenção, uma vez que o cônjuge pode fazer parte das etapas da doença, incluindo a suspeita, o diagnóstico, o tratamento e, consequentemente, o prognóstico. Em 2006, Maia e Basso, num estudo no Estado de São Paulo verificaram que o cônjuge foi responsável por perceber a lesão suspeita de MC numa importante parcela dos casos, principalmente no grupo dos homens, onde $29 \%$ tiveram suas lesões detectadas por suas esposas. ${ }^{23}$ Este dado pode explicar a proteção conferida pela presença de parceiro no sexo masculino.

O predomínio de baixo nível de escolaridade encontrado neste estudo pode estar diretamente relacionado à mortalidade no MC. Segundo a literatura, existe melhor prognóstico do melanoma em pacientes com maior grau de escolaridade (maior conhecimento, maior acesso à informação), fator que é associado ao diagnóstico precoce. ${ }^{24}$ Entretanto, neste estudo, a maior escolaridade mostrou-se fator de proteção apenas no sexo feminino. Isto pode se justificar na maior preocupação das mulheres com a saúde e nas diferenças comportamentais entre os sexos já citadas anteriormente.

O presente estudo vem contribuir para uma melhor compreensão do MC e também para nortear médicos especialistas e generalistas a lidar com esses pacientes e a conduzir campanhas de prevenção que resultem em uma maior conscientização da população, consequentemente favorecendo o diagnóstico precoce, fundamental no prognóstico do MC.

Uma possível limitação desse estudo pode residir na inconsistência do banco de dados (DATASUS) quanto à quantidade (como o preenchimento incompleto), qualidade (falta de variáveis importantes para avaliação do comportamento do tumor, como estadiamento) e processamento das informações de óbito por MC. Porém, a análise de um período de dez anos acreditamos minimizar possíveis deficiências do sistema.
Conflitos de interesse: Os autores declaram não possuir conflitos de interesse.

Suporte financeiro: $O$ presente trabalho não foi suportado por nenhum subsídio ou bolsa.

Confidencialidade dos dados: Os autores declaram ter seguido os protocolos do seu centro de trabalho acerca da publicação dos dados de doentes.

Direito a privacidade e consentimento escrito: Os autores declaram que pediram consentimento ao representante legal para usar as imagens no artigo.

Protecção de pessoas e animais: Os autores declaram que os procedimentos seguidos estavam de acordo com os regulamentos estabelecidos pelos responsáveis da Comissão de lnvestigação Clínica e Ética e de acordo com a Declaração de Helsínquia da Associação Médica Mundial

Conflicts of interest: The authors have no conflicts of interest to declare.

Financing Support: This work has not received any contribution, grant or scholarship.

Confidentiality of data: The authors declare that they have followed the protocols of their work center on the publication of data from patients.

Privacy policy and informed consent: The authors declare that the legal representative of the patient gave written informed consente for the use of patient's photos in this article.

Protection of human and animal subjects: The authors declare that the procedures followed were in accordance with the regulations of the relevant clinical research ethics committee and with those of the Code of Ethics of the World Medical Association (Declaration of Helsinki).

\section{REFERÊNCIAS}

1. Shain AH, Yeh I, Kovalyshyn I, Sriharan A, Talevich E, Gagnon $A$, et al. The genetic evolution of melanoma from precursor lesions. New Engl J Med. 2015; 373: 1926-36. doi: 10.1056/NEJMoa1502583.

2. World Health Organization. Ultraviolet radiation and the INTERSUN Programme [Internet]. [acesso em $27 \mathrm{abr}$ 2014]. Disponível em: http://www.who.int/uv/faq/skincancer/en/index 1.html.

3. Marks R. Epidemiology of melanoma. Clin Exp Dermatol. 2000; 25: 459-63.

4. Demográfico, IBGE Censo. "Fundação Seade (Sistema Estadual de análise de Dados)" [Acesso em maio 2018] Disponível em: http://www.seade.gov.br/

5. Bonfá R, Bonamigo RR, Bonfá R, Duro KM, Furian RD, Zelmanowicz AM. A precocidade diagnóstica do melanoma cutâneo: uma observação no sul do Brasil. An Bras Dermatol. 2011 ; 86:215-21.

6. Whiteman DC, Green AC, Olsen CM. The growing burden of invasive melanoma: projections of incidence rates and numbers of new cases in six susceptible populations through 2031. J Investig Dermatol. 2016 ; 136:1161-71. doi: 10.1016/i.jid.2016.01.035.

7. Coory M, Baade P, Aitken J, Smithers M, McLeod GR, Ring I. Trends for in situ and invasive melanoma in Queensland, Australia, 1982-2002. Cancer Causes Control. 
$2006 ; 17: 21-7$.

8. Karimkhani C, Green AC, Niijsten T, Weinstock MA, Dellavalle RP, Naghavi, M,et al. The global burden of melanoma: results from the Global Burden of Disease Study 2015. Br J Dermatol. 2017; 177: 134-40.

9. Instituto Nacional de Câncer José de Alencar Gomes da Silva [homepage na internet]. Tipos de Câncer: Pele: Melanoma. [acesso em 22/03/2018]. Disponível em: http:// www2.inca.gov.br

10. Ferreira FR, Nascimento LF. Mortality due to cutaneous melanoma in south region of Brazil: a spatial approach. An Bras Dermatol. 2016; 91:437-41.

11. Ferrari Júnior $N M$, Muller $H$, Ribeiro $M$, Maia $M$, Sanches Júnior JA. Cutaneous melanoma: descriptive epidemiological study. Sao Paulo Med J. 2008; 126:41-7.

12. Departamento de Informática do SUS - DATASUS. Estatísticas Vitais. [Acesso em mar 2018] Disponível em: http://www.datasus.gov.br

13. Stata Corporation. Statistical software for professionals/ STATA [programa de computador]. Versão 9. Texas: College Station; 2005.

14. Tuong, William, Lily S. Cheng, and April W. Armstrong. "Melanoma: epidemiology, diagnosis, treatment, and outcomes." Dermatol Clin. 2012; 30: 113-24.

15. Leiter U, Garbe C. Epidemiology of melanoma and nonmelanoma skin cancer-the role of sunlight. Adv Exp Med Biol. 2008; 624:89-103. doi: 10.1007/978-0-38777574-6_8.

16. Nikolaou V, Stratigos AJ. Emerging trends in the epidemiology of melanoma. Br J Dermatol. 2014; 170: 11-9.
17. Pinto AC, Cavalcante ML, da Silva GV, de Brito FF, Oliveira AM, Cleto NG. Melanoma maligno: estudo epidemiológico dos casos diagnosticados em unidade de referência em dermatologia em Bauru-sp de 2007 a 2014. Surg Cosmetic Dermatol. 2015; 7: 104-7.

18. Martins-Costa GM, Bonamigo RR, Menegat AP, Costa LL, Bonfá R, Grazziotin TC. Melanoma cutâneo primário: confirmações e novidades. Rev AMRIGS. 2013; 57: 20812.

19. Ferreira FR, Nascimento LF. Mortality due to cutaneous melanoma in south region of Brazil: a spatial approach. An Brasil Dermatol. 2016; 91:, 437-41.

20. Mikkilineni R, Weinstock MA. Epidemiology. In: Sober A, Haluska F, editors. Skin cancer. London: BC Decker; 2001. p. 1-15.

21. Cormier JN, Xing Y, Ding M, Lee JE, Mansfield PF, Gershenwald JE, et al. Ethnic differences among patients with cutaneous melanoma. Arch Intern Med. 2006; 166:190714. doi: 10.1001/archinte.166.17.1907.

22. Hu S, Parmet $Y$, Allen G, Parker DF, Ma F, Rouhani P, et al. Disparity in melanoma: a trend analysis of melanoma incidence and stage at diagnosis among whites, Hispanics, and blacks in Florida. Arch Dermatol. 2009;145:136974. doi: 10.1001/archdermatol.2009.302.

23. Maia M, Basso M. Quem descobre o melanoma cutâneo? An Bras Dermatol. 2006;81:244-8.

24. Mackie RM, Hole DJ. Incidence and thickness of primary tumours and survival of patients with cutaneous malignant melanoma in relation to socioeconomic status. BMJ. 1996;312:1125-8. 